\title{
A Dynamic Control Law for the Wave Equation*
}

\author{
ÖMER MORGÜL†
}

Key Words-Distrubuted parameter systems; partial differential equations; boundary value problems; stability; Lyapunov methods.

\begin{abstract}
We consider a system described by the onedimensional linear wave equation in a bounded domain with appropriate boundary conditions. To stabilize the system, we propose a dynamic boundary controller applied at the free end of the system. The transfer function of the proposed controller is restricted to be a positive real function which could be strictly proper. We then show that, if the transfer function of the controller is strictly proper, then the resulting closed-loop system is asymptotically stable, and if proper but not strictly proper, then the resulting closed-loop system is exponentially stable.
\end{abstract}

\section{Introduction}

MANY MECHANICAL systems, such as spacecraft with flexible attachments, or robots with flexible links, and many practical systems such as power systems or mass transport systems contain certain parts whose dynamic behaviour can be rigorously described only by partial differential equations (PDE). In such systems, to achieve high precision demands, the dynamic effect of the system parts, whose behaviour is described by PDE's, on the overall system has to be taken into account in designing the controllers.

In recent years, boundary control of systems represented by PDE's has become an important research area. This idea was first applied to the systems represented by the wave equation (e.g. elastic strings, cables), see Slemrod (1976), Chen (1979), Lagnese (1983), and recently extended to the beam equations, see Chen et al. $(1987 \mathrm{a}, \mathrm{b})$, and to the rotating flexible structures, see Morgul $(1990,1991 \mathrm{a}, \mathrm{b})$. In particular, it has been shown that for a string which is clamped at one end and is free at the other end, a single non-dynamic boundary control applied at the free end is sufficient to exponentially stabilize the system, see Chen (1979) and Lions (1988). Control problems for the wave equation have been discussed by very many scientists, and to mention only a few we note the works of Lasiecka and Triggiani (1987), Datko (1988), Lasiecka (1989), Komornik and Zuazua (1990), Bardos et al. (1992) and Conrad and Rao (1993). A good source of references to papers in which boundary stabilization problems are treated can be found in Lagnese (1989). See also Lions (1988).

In this note, we consider a linear time-invariant system which is represented by a one-dimensional wave equation in a bounded domain. We assume that the system is clamped at one end and the boundary control input is applied at the other end. For this system, we propose a finite-dimensional dynamic boundary controller. This introduces extra degrees of freedom in designing controllers which could be exploited

\footnotetext{
* Received 1 December 1992; revised 7 May 1993; revised 1 October 1993; received in final form 6 December 1993. This paper was not presented at any IFAC meeting. This paper was recommended for publication in revised form by Associate Editor R. F. Curtain under the direction of Editor Huibert Kwakernaak. Corresponding author Professor Ö. Morgul. Tel. +90 $3122664000 / 1529$; Fax +90 312266 4126; E-mail morgul@ee.bilkent.edu.tr \& morgul@trbilun.bitnet.

† Department of Electrical and Electronics Engineering, Bilkent University, 06533 Bilkent, Ankara, Turkey.
}

in solving a variety of control problems, such as disturbance rejection, pole assignment, etc. while maintaining stability. The transfer function of the controller is restricted to be a positive real function. This class of controllers is quite large and contains some previously proposed non-dynamic controllers as a special case. We show that, if the controller transfer function is strictly proper, the resulting closed-loop system is asymptotically, but not exponentially stable. The main advantage of this type of controller is that the resulting open-loop map is also strictly proper, which is important in proving certain stability robustness results (Helmicki et al. (1991)). On the other hand, if the controller transfer function is proper but not strictly proper, we show that the resulting closed-loop system is exponentially stable. We also present some simulation results in case the controller output is corrupted by a disturbance.

\section{Problem statement}

We consider a string as an example of a system whose behaviour is modelled by the wave equation. Without loss of generality, we assume that the string length, mass density and the string tension are as $L=1, \rho=1$ and $T=1$, respectively. We denote the displacement of the string by $y(x, t)$ at $x \in(0,1)$ and $t \geq 0$. Furthermore, we assume that the string is fixed at one end and stabilized by dynamic boundary control at the other end. Thus, the system under consideration is represented by:

$$
\begin{gathered}
y_{n}(x, t)=y_{x x}(x, t), \quad x \in(0,1), \quad t \geq 0 \\
y(0, t)=0, \quad t \geq 0 \\
y_{x}(1, t)=-f(t), \quad t \geq 0,
\end{gathered}
$$

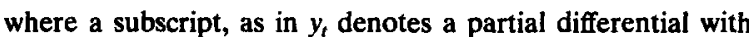
respect to the corresponding variable, and $f(\cdot): \mathbf{R}_{+} \rightarrow \mathbf{R}$ is the boundary control force applied at the free end of the string. We note that the systems represented by (1)-(3) are not restricted to strings; for example vibrations of long cables, the longitudinal motion and the torsional vibrations of elastic beams can also be represented by these equations, see e.g. Meirovitch (1967). We assume that $f(t)$ is generated by the following dynamic actuator:

$$
\begin{gathered}
\dot{w}(t)=A w(t)+b y_{,}(1, t), \quad t \geq 0 \\
f(t)=c^{\mathbf{T}} w(t)+d y_{t}(1, t)+k y(1, t), \quad t \geq 0,
\end{gathered}
$$

where $w \in \mathbf{R}^{n}$, for some natural number $n$, is the actuator state, $A \in \mathbf{R}^{n \times n}$ is a constant matrix, $b, c \in \mathbf{R}^{n}$ are constant column vectors, $d, k$ are constant real numbers and the superscript $T$ denotes transpose.

Remark 1. In the case of a non-dynamic actuator, (4) will not exist, and (5) will reduce to

$$
f(t)=d y_{f}(1, t)+k y(1, t), \quad t \geq 0,
$$

which is the case considered in Chen (1979). Therefore, the actuator proposed above may be considered as a generalization of the control law given by (6), see Slemrod (1976), Chen (1979), Lagnese (1983).

It is known that if the boundary control force is set to zero 
(i.e. $f \equiv 0$ ), then the system given by (1)-(3) has an infinite number of eigenvalues on the imaginary axis, see e.g. Meirovitch (1967). Our problem is to design an actuator given by (4), (5) such that the resulting system given by (1)-(5) is stable in some sense.

The stabilization problem stated above can be solved by means of a non-dynamic actuator. For example, in Chen (1979) it was proven that the system given by (1)-(3) and (6) is exponentially stable provided that $d>0$ and $k \geq 0$. However, the actuator transfer function given by (6) is not strictly proper, which might cause some problems in actual implementation, see Helmicki et al. (1991). It is clear that in order to solve a variety of control problems, control laws more general than (6) are required. The main motivation of this paper is to propose a large class of finite-dimensional stabilizing controllers for the system given by (1)-(3). The proposed dynamic controller given by (4), (5) is a candidate for such a class which also covers the controller given by (6).

We also note that the proposed controller offers an extra degree of freedom in designing the controllers. This extra degree of freedom could be exploited to solve a variety of control problems, such as eigenvalue assignment, disturbance rejection etc., while maintaining stability, see Morgül (1992b). Preliminary simulation studies show that by using the controller given by (4), (5), it could be possible to change the eigenvalues of the system given by (1)-(5) over a specified frequency range, without affecting the rest of the spectrum very much, see Morgull (1992b). We note that this could not be achieved by using the controller given by (6) since in this case the spectrum of the system is affected uniformly.

\section{Stability results}

We first make the following assumptions concerning the actuator given by (4), (5) thoroughout this work

Assumption 1. All eigenvalues of $A \in \mathbf{R}^{n \times n}$ have negative real parts.

Assumption 2. $(A, b)$ is controllable and $(c, A)$ is observable.

Assumption 3. $d \geq 0, k \geq 0$; moreover there exists a constant $\gamma, d \geq \gamma \geq 0$, such that the following holds:

$$
d+\mathscr{R} e\left\{c^{\top}(j \omega I-A)^{-1} b\right\}>\gamma, \quad \omega \in \mathbf{R} .
$$

We define the transfer function $g(s)$ of the actuator given by (4), (5) as:

$$
g(s)=d+c^{\mathrm{T}}(s I-A)^{-1} b+\frac{k}{s}
$$

If we take the Laplace transform in (4) and (5) and use zero initial conditions, we obtain:

$$
\hat{f}(s)=g(s) \hat{y}_{i}(1, s),
$$

where a hat denotes the Laplace transform of the corresponding variable. This, together with (7) implies that the transfer function in (8) is a positive real function, and the transfer function $g_{1}(s)=g(s)-k / s$ is a strictly positive real transfer function, see Slotine and $\mathrm{Li}$ (1991, pp. 130-131).

If the actuator is given by a transfer function as follows:

$$
\hat{f}(s)=g(s) \hat{y}_{i}(1, s),
$$

then we see that $k$ in (8) is the residue of the pole of $g(s)$ at $s=0$. Let $g_{1}(s)=g(s)-k / s$. One can always find a minimal realization $(A, b, c, d)$ for $g_{1}(s)$, and because of minimality, the eigenvalues of $A$ are the same as the poles of $g_{1}(s)$ Hence, in case the actuator is given by a proper transfer function $g(s)$, as in $(10)$, as opposed to the state-space representation given by (4), (5), an equivalent characterization of the Assumptions 1-3 are:

Assumption 4. $g(s)$ has at most a simple pole at $s=0$ and the residue associated with this pole is non-negative; all remaining poles of $g(s)$ have negative real parts.

Assumption 5. There exists a $\gamma \geq 0$ such that the following holds:

$$
\mathscr{R e}\{g(j \omega)\}>\gamma, \quad \omega \in \mathbf{R} .
$$

Let Assumptions 1-3, or equivalently Assumptions 4-5 stated above hold. Then, since the transfer function $g_{1}(s)=d+c^{\mathrm{T}}(s I-A)^{-1} b$ is strictly positive real it follows from the Meyer-Kalman-Yakubovich lemma that given any symmetric positive definite matrix $Q \in \mathbf{R}^{n \times n}$, there exists a symmetric positive definite matrix $P \in \mathbf{R}^{n \times n}$, a vector $q \in \mathbf{R}^{n}$ and a constant $\epsilon>0$ satisfying:

$$
\begin{gathered}
A^{\mathrm{T}} P+P A=-q q^{\mathrm{T}}-\epsilon Q \\
P b-c=\sqrt{2(d-\gamma)} q,
\end{gathered}
$$

see Slotine and $\mathrm{Li}(1991$, p. 133)

To analyse the system given by (1)-(5), we first define the function space $\mathscr{H}$ as follows:

$$
\left.\mathscr{H}:=\left\{\begin{array}{lll}
u & v & w
\end{array}\right)^{\mathrm{T}} \mid u \in \mathbf{H}^{1}, v \in \mathbf{L}^{2}, w \in \mathbf{R}^{n}, u(0)=0\right\},
$$

where the spaces $L^{2}$ and $H^{k}$ are defined as follows:

$$
\begin{aligned}
\mathbf{L}^{2} & =\left\{f:[0, L] \rightarrow \mathbf{R} \mid \int_{0}^{L} f^{2} \mathrm{~d} x<\infty\right\} \\
\mathbf{H}^{k} & =\left\{f \in \mathbf{L}^{2} \mid f, f^{\prime}, f^{\prime \prime}, \ldots, f^{(k)} \in \mathbf{L}^{2}\right\} .
\end{aligned}
$$

The equations (1)-(5) can be written in the following abstract form:

$$
\dot{z}=L z, \quad z(0) \in \mathscr{K},
$$

where $z=\left(\begin{array}{lll}y & y & w\end{array}\right)^{\mathrm{T}} \in \mathscr{H}$, the operator $L: \mathscr{H} \rightarrow \mathscr{H}$ is a linear unbounded operator defined as

$$
L\left(\begin{array}{c}
u \\
v \\
w
\end{array}\right)=\left(\begin{array}{c}
v \\
u_{x x} \\
A w+b v(1)
\end{array}\right) .
$$

The domain $D(L)$ of the operator $L$ is defined as:

$$
\begin{aligned}
D(L):= & \left\{\left(\begin{array}{lll}
u & v & w
\end{array}\right)^{\mathrm{T}} \mid u \in \mathbf{H}^{2}, v \in \mathbf{H}^{1}, w \in \mathbf{R}^{n}, u(0)=0\right. \\
& \left.v(0)=0 ; u_{x}(1)+c^{\mathrm{T}} w+d v(1)+k u(1)=0\right\} .
\end{aligned}
$$

Let Assumptions 1-3 hold, let $Q \in \mathbf{R}^{n \times n}$ be an arbitrary symmetric positive definite matrix and let $P \in \mathbf{R}^{n \times n}, q \in \mathbf{R}^{n}$ be the solutions of (12) and (13), where $P$ is also a symmetric and positive definite matrix. In $\mathscr{H}$, we define the following 'energy' inner-product:

$$
\langle z, \tilde{z}\rangle_{E}=\frac{1}{2} \int_{0}^{1} y_{t} \tilde{y}_{t} \mathrm{~d} x+\frac{1}{2} \int_{0}^{1} y_{x} \tilde{y}_{x} \mathrm{~d} x+\frac{1}{2} k y(1) \tilde{y}(1)+\frac{1}{2} \tilde{w}^{\mathrm{T}} P w,
$$

where $z=\left(\begin{array}{lll}y & y_{t} & w\end{array}\right)^{\mathrm{T}}, \tilde{z}=\left(\begin{array}{lll}y & \tilde{y}_{t} & \tilde{w}\end{array}\right)^{\mathrm{T}}$. It is well-known that the first two integral terms represent an inner product for $\mathbf{H}^{1} \times \mathbf{L}^{2}$, see e.g. Chen (1979). By using (2), the term multiplied by $k$ can be embedded in the second integral, see (22) below. Since the last term gives an inner product for $\mathbf{R}^{n}$, it follows that $\mathscr{H}$, together with the energy inner-product given by (20) becomes a Hilbert space. The 'energy' norm induced by $(20)$ is:

$$
\begin{aligned}
E(t): & =\|z(t)\|_{E}^{2} \\
& =\frac{1}{2} \int_{0}^{1} y_{t}^{2} \mathrm{~d} x+\frac{1}{2} \int_{0}^{1} y_{x}^{2} \mathrm{~d} x+\frac{1}{2} k y^{2}(1, t)+\frac{1}{2} w^{\mathrm{T}} P w .
\end{aligned}
$$

In the sequel we need the following inequality which follows from Jensen's inequality, Royden $(1968$, p. 110):

$$
y^{2}(x) \leq \int_{0}^{x}\left(y^{\prime}\right)^{2} \mathrm{~d} s \quad \forall x \in[0,1] \quad \forall y \in \mathbf{H}^{1}, \quad y(0)=0 .
$$

Theorem 1 . Consider the system given by (17), where the operator $L$ is given by (18). Then

(i) the operator $L$ generates a $C_{0}$-semigroup of contractions $T(t)$ on $\mathscr{H}$

(ii) the $C_{0}$-semigroup $T(t)$ generated by $L$ is 
asymptotically stable, that is the energy given by (21) asymptotically tends to zero along the solutions of (17).

Proof. (i) We use Lumer-Phillips theorem, see Pazy (1983, p. 14) to prove the assertion (i). To prove that $L$ is dissipative, we differentiate (21) with respect to time. Then by using (1)-(5), integrating by parts and using (12), (13), we obtain:

$$
\begin{aligned}
\dot{E}= & \int_{0}^{1} y_{t} y_{t} \mathrm{~d} x+\int_{0}^{1} y_{x} y_{x t} \mathrm{~d} x+\frac{1}{2}\left(\dot{w}^{\mathrm{T}} P w+w^{\mathrm{T}} P \dot{w}\right) \\
& +k y(1, t) y_{t}(1, t) \\
= & -y_{t}(1, t) f(t)+\frac{1}{2} w^{\mathrm{T}}\left(A^{\mathrm{T}} P+P A\right) w \\
& +w^{\mathrm{T}} P b y_{t}(1, t)+k y(1, t) y_{t}(1, t) \\
= & -c^{\mathrm{T}} w y_{t}(1, t)-\mathrm{d} y_{t}^{2}(1, t)+w^{\mathrm{T}} P b y_{t}(1, t) \\
& -\frac{1}{2} w^{\mathrm{T}} q q^{\mathrm{T}} w-\frac{\epsilon}{2} w^{\mathrm{T}} Q w \\
= & -\gamma y_{t}^{2}(1, t)-\frac{1}{2}\left[\sqrt{2(d-\gamma)} y_{t}(1, t)-w^{\mathrm{T}} q\right]^{2}-\frac{\epsilon}{2} w^{\mathrm{T}} Q w,
\end{aligned}
$$

where to obtain the first equation, we differentiated (21) with respect to time, to obtain the second equation we used (1), (2), (4), and integration by parts, to obtain the third equation we used (5) and (12), and finally to obtain the last equation, we used (13). Since $\dot{E} \leq 0$, it follows that $L$ is dissipative [see (20), (21), (23)].

Hence, to prove assertion (i), it is enough to show that for some $\lambda>0$, the operator $\lambda I-L: \mathscr{H} \rightarrow \mathscr{H}$ is onto. Let $(f h r)^{\mathrm{T}} \in \mathscr{K}$ be given. We have to find $(u v w)^{\mathrm{T}} \in D(L)$ such that:

$$
\begin{gathered}
\lambda u-v=f \\
\lambda v-u_{x x}=h \\
\lambda w-A w-b v(1)=r \\
u(0)=0, \quad u_{x}(1)+c^{\mathrm{T}} w+d v(1)+k u(1)=0
\end{gathered}
$$

for some $\lambda>0$

Using (24) in (25), we obtain:

$$
\lambda^{2} u-u_{x x}=h+\lambda f,
$$

whose solution satisfying $u(0)=0$ is given by:

$$
u(x)=c_{1} \sinh \lambda x-\frac{1}{\lambda} \int_{0}^{x}(h(s)+\lambda f(s)) \sinh \lambda(x-s) \mathrm{d} s
$$$$
x \in(0,1)
$$

where $c_{1}$ is a constant and $\sinh (\cdot)$ is the hyperbolic sine function. By using (24) in (26) we find $w$ in terms of $u(1)$ as follows:

$$
w=(\lambda I-A)^{-1}(\lambda u(1) b+r-f(1) b) .
$$

By using (24), (29) and (30) in (27), we obtain:

$\lambda(\cosh \lambda+g(\grave{\lambda}) \sinh \lambda) c_{1}$

$$
\begin{aligned}
=\int_{0}^{1}(h(s)+\lambda f(s))(\cosh \lambda(1-s)+g(\lambda) \sinh \lambda(1-s)) \mathrm{d} s \\
+\left(g(\lambda)-\frac{k}{\lambda}\right) f(1)-c^{\mathrm{T}}(\lambda I-A)^{-1} r,
\end{aligned}
$$

where $g(\lambda)$ is the actuator transfer function given by (8). Since $g(s)$ is a positive real function it follows that $g(\lambda)>0$ for all $\lambda>0$, see e.g. Slotine and $\mathrm{Li}$ (1991, pp. 129-130); moreover $\lim g(\lambda)=d \geq 0$, see (8). It then follows that

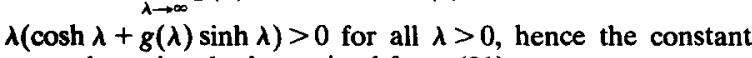
$c_{1}$ can be uniquely determined from (31) as:

$$
\begin{aligned}
& \int_{0}^{1}(h(s)+\lambda f(s))(\cosh \lambda(1-s) \\
&+g(\lambda) \sinh \lambda(1-s)) \mathrm{d} s \\
&+\left(g(\lambda)-\frac{k}{\lambda}\right) f(1)-c^{\mathrm{T}}(\lambda I-A)^{-1} r
\end{aligned}
$$

The remaining unknowns $v$ and $w$ can be found from (24) and (30), respectively. It can easily be shown that $\left(\begin{array}{lll}u & v & w\end{array}\right)^{\mathrm{\top}} \in D(L)$. This proves that for all $\lambda>0$, the operator $\lambda I-L: \mathscr{H} \rightarrow \mathscr{H}$ is onto. Since $\mathscr{H}$ is a Hilbert space, it follows that $D(L)$ is dense in $\mathscr{H}$, see Pazy $(1983$, p. 16$)$. By the Lumer-Phillips theorem, it follows that $L$ generates a $C_{0}$-semigroup of contractions $T(t)$ on $\mathscr{H}$

(ii) To prove the assertion (ii), we use LaSalle's invariance principle, extended to infinite-dimensional systems, see Saperstone (1981, p. 78) and Hale (1969). According to this principle, all solutions of (17) asymptotically tend to the maximal invariant subset of the following set:

$$
\mathscr{S}=\{z \in \mathscr{H} \mid \dot{E}=0\},
$$

provided that the solution trajectories for $t \geq 0$ are precompact in $\mathscr{H}$. Since the operator $L: \mathscr{H} \rightarrow \mathscr{H}$ generates a $C_{0}$-semigroup of contractions on $\mathscr{H}$ (hence the solution trajectories are bounded on $\mathscr{H}$ for $t \geq 0$ ), the precompactness of the solution trajectories are guaranteed if the operator $(\lambda I-L)^{-1}: \mathscr{H} \leftarrow \mathscr{H}$ is compact for some $\lambda>0$, see Dafermos and Slemrod (1973) and Saperstone (1981, p. 241). To prove the last property, we first show that $L^{-1}$ exists and is a compact operator on $\mathscr{H}$. To see this, we put $\lambda=0$ in (24)-(27), which results in the following solution $(u v w)^{\mathrm{T}} \in$ $\mathscr{H}$ for any given $(f h r)^{\mathrm{T}} \in \mathscr{H}$ :

$$
\begin{gathered}
u(x)=-\int_{0}^{x} \int_{0}^{\xi} h(\sigma) \mathrm{d} \sigma \mathrm{d} \xi+c_{2} x \quad x \in(0,1) \\
v(x)=-f(x), \quad x \in(0,1) \\
w=A^{-1}(f(1) b-r),
\end{gathered}
$$

where the constant $c_{2}$ can be uniquely determined from (27) It follows that $L^{-1}$ exists and maps $\mathscr{H}$ into $\mathbf{H}^{2} \times \mathbf{H}^{1} \times \mathbf{R}^{n}$. moreover $(u v w)^{\mathrm{T}} \in D(L)$. Since $(f h r)^{\mathrm{T}} \in \mathscr{H}$ it follows that $f(0)=0$ and that $f \in \mathbf{H}^{1}$, see (14). Hence, if $\left\|(f h r)^{\mathbf{T}}\right\|$ is bounded in $\mathscr{H}$, it follows from (21) and (22) that $f(1)$ is bounded as well. Therefore $L^{-1}$ maps the bounded sets of $\mathscr{H}$ into the bounded sets of $\mathbf{H}^{2} \times \mathbf{H}^{1} \times \mathbf{R}^{n}$. Since the embedding of the latter into $\mathscr{H}$ is compact, see Tanabe $(1979$, p. 14$)$, it follows that $L^{-1}$ is a compact operator. This also proves that the spectrum of $L$ consists entirely of isolated eigenvalues, and that for any $\lambda$ in the resolvent set of $L$, the operator $(\lambda I-L)^{-1}: \mathscr{H} \rightarrow \mathscr{H}$ is a compact operator, see Kato $(1980, \mathrm{p}$. 187). Furthermore, our argument above shows that $\lambda=0$ is not an eigenvalue of $L$. Since the operator $L$ generates a $C_{0}$-semigroup of contractions on $\mathscr{H}$, by the argument given above it follows that the solutions trajectories of (17) are precompact in $\mathscr{H}$ for $t \geq 0$, hence by LaSalle's invariance principle, the solutions asymptotically tend to the maximal invariant subset of $\mathscr{S}$ [see (33)]. Hence, to prove that all solutions of (17) asymptotically tend to the zero solution, it suffices to show that $\mathscr{P}$ contains only the zero solution, which is a typical procedure in the application of LaSalle's invariance principle, see e.g. Saperstone (1981).

To prove that $\mathscr{S}$ contains only the zero solution, we set $\dot{E}=0$ in (23), which results in $w=0$. This implies that $\dot{w}=0$, hence by using (4) and (5) we obtain $y_{t}(1, t)=0$, $f(t)=k y(1, t)$. Since all boundary conditions are separable the solution of (1) can be found by using separation of variables, see Meirovitch (1967). That is, the solution of (1) and (2) with the following boundary conditions:

$$
y_{t}(1, t)=0, \quad y_{x}(1, t)+k y(1, t)=0
$$

assumes the following form

$$
y(x, t)=A(t) B(x), \quad t \geq 0, x \in[0,1]
$$

where the functions $A: \mathbf{R}_{+} \rightarrow \mathbf{R}$ and $B:[0,1] \rightarrow \mathbf{R}$ are twice differentiable functions to be determined from the boundary conditions (2) and (37). We distinguish two cases: 
(a) $A \equiv 0$. In this case, from (1) it follows that $B(x)=c_{1}+c_{2} x$, where $c_{1}$ and $c_{2}$ are arbitrary constants. By using this result in (2) and (37), we obtain $c_{1}=0$ and $(1+k) c_{2}=0$, which implies $c_{2}=0$, since $k \geq 0$. This shows that $y(x, t) \equiv 0$.

(b) $\dot{A} \neq 0$. In this case, by using (38) in (1), we obtain the following solution for $B$ :

$$
B(x)=c_{1} \cos \beta x+c_{2} \sin \beta x,
$$

where $c_{1}, c_{2}$ and $\beta$ are to be determined from (2) and (37). By using (2) we obtain $c_{1}=0$ and by using (37) we obtain $c_{2} \sin \beta=0$ and $c_{2} \beta \cos \beta=0$ which shows that either $c_{2}=0$ or $\beta=0$; but in both cases we have $B(x) \equiv 0$, hence $y(x, t) \equiv 0$. We note that the same result could be obtained by using Fourier analysis, see d'Andrea-Novel et al. (1990) for a similar result.

Therefore we conclude that the only solution of (17) which lies in the set $\mathscr{S}$ given by (33) is the zero solution. Hence, by LaSalle's invariance principle, we conclude that the solutions of (17) asymptotically tend to the zero solution.

Remark 2. Let us investigate the system given by (1)-(5) from an input/output point of view. Let us define the boundary control force $f(t)$ as the input and $y_{t}(1, t)$ as the output. If we take the Laplace transform of (1), use zero initial conditions, and use the boundary conditions (2), (3), we obtain the plant transfer function $p(s)$ as $p(s)=$ $-\sinh s / \cosh s$. Let $\mathbf{C}$ denote the set of complex numbers, and for $\sigma \in \mathbf{R}$ let $\mathbf{C}_{\sigma^{+}}=\{s \in \mathbf{C} \mid \operatorname{Re}(s) \geq \sigma\}$. Although $p(s)$ is not bounded on the imaginary axis, it is bounded on $\mathbf{C}_{\sigma^{+}}$ for any $\sigma>0,\left[p \in A_{-}(\infty)\right.$ with the notation of Helmicki $e t$ al. (1991), see also Callier and Wilkin (1986)]. Let us take $d=0$ in the controller given by (5), which results in a strictly proper compensator $g(s)$, see (8). The resulting open-loop map $g(s) p(s)$ is also strictly proper in the sense that for any $\sigma>0, g(s) p(s) \rightarrow 0$ as $|s| \rightarrow \infty, s \in \mathbf{C}_{\sigma^{+}}$, see Helmicki et al. (1991). Hence, for the system given by (1)-(3), there exist strictly proper controllers for which the open-loop map is also strictly proper and the closed-loop system is stable in an asymptotic sense. However, if the stability is understood in an exponential sense and if it is also required that the compensator transfer function and/or open-loop map be strictly proper following Helmicki et al. (1991, Corollary 4.1), it can be shown that for the system given by (1)-(3) such a controller does not exist [for more details see Helmicki (1988, p. 47)]. This shows that for infinite dimensional systems, exponential stability requirement may be too restrictive if we also require strictly proper stabilizing compensators and/or strictly proper open-loop maps, and that asymptotic stability requirement may be more suitable for designing such controllers. This point requires further research.

The above argument shows that when $d=0$, that is the controller given by (10) is strictly proper, the resulting closed-loop system cannot be exponentially stable. In the following we prove that if the controller is proper but not strictly proper, (i.e. $d>0$ ), exponential stability may be obtained. To prove this result, we use the following theorem.

Theorem 2. Let $T(t)$ be a $C_{0}$-semigroup generated by a linear operator $L$ in a Hilbert space. Assume that $T(t)$ satisfies:

$$
\|T(t)\| \leq B, \quad t \geq 0
$$

for some $B>0$. Then, there exist $M>0$ and $\delta>0$ such that:

$$
\|T(t)\| \leq M e^{-\delta t}, \quad t \geq 0
$$

if and only if the imaginary axis belongs to the resolvent set of $L$, and

$$
\sup _{\omega \in \mathbf{R}}\left\|(j \omega I-L)^{-1}\right\|<\infty
$$

Proof. See Huang (1985). This theorem is attributed in the literature to F. Huang, see e.g. Chen et al. (1987b). We note that the same result was also given in Pruss (1984, Corollary 4). See also Gearhart (1978) for the same result but limited to $C_{0}$-contraction semigroups.

For an application of this theorem to flexible structures, see Chen et al. (1987b).

Theorem 3. Consider the system given by (17). Let the controller given by (4) and (5) satisfy (7) with $d \geq \gamma>0$, (cf. with Assumption 3). Then, the semigroup $T(t)$ generated by the operator $L$ given by (18) is exponentially stable.

Remark 3. The main distinction between Theorem 3 and Theorem 1 is that, in Theorem 1, the real part of the transfer function, $\mathscr{R} e(g(j \omega))$ is required to be strictly positive, whereas in Theorem 3, it is required to be bounded away from zero.

Remark 4. For the non-dynamic case, i.e. when the controller is given by (6) with $d>0, k \geq 0$, instead of (4), (5), the exponential stability was proven in Chen (1979). Hence, the result presented here may be considered as a generalization of that result. However, we note that the techniques we use in the following proof are entirely different than those employed in Chen (1979).

Proof. By Theorem 1, the operator $L$ generates a $C_{0}$-semigroup $T(t)$ on $\mathscr{H}$. By (23) this semigroup is bounded, i.e. (39) is satisfied. Also, by Theorem 1, the spectrum of $L$ consists entirely of many countable isolated eigenvalues, and $\lambda=0$ is not an eigenvalue of $L$.

Next we prove by contradiction that the imaginary axis belongs to the resolvent set of $L$. Suppose that the spectrum of $L$ and the imaginary axis have common points. Since the operator $L$ has a point (i.e. discrete) spectrum, it follows that there exists an $\omega \in \mathbf{R}$ such that $(24)-(27)$ has a nontrivial solution for $\lambda=j \omega$ and $(f h r)^{\mathrm{T}}=\left(\begin{array}{lll}0 & 0 & 0\end{array}\right)^{\mathrm{T}}$. This solution is given as:

$$
\begin{gathered}
u(x)=j c_{1} \sin \omega x, \quad v(x)=-\omega c_{1} \sin \omega x \\
w=-c_{1} \omega \sin \omega(j \omega l-A)^{-1} b, \quad x \in(0,1)
\end{gathered}
$$

where $c_{1}$ is an arbitrary constant [see (29), (24) and (30)]. By putting $\lambda=j \omega$ and $\left(\begin{array}{ll}f h r\end{array}\right)^{\mathrm{T}}=\left(\begin{array}{lll}0 & 0 & 0\end{array}\right)^{\mathrm{T}}$ in $(31)$, the latter becomes:

$$
c_{1}[j \omega \cos \omega-\omega g(j \omega) \sin \omega]=0,
$$

where $g(s)$ is given by $(8)$. Let $R(\omega)$ and $I(\omega)$ be the real and imaginary parts of $g(j \omega)$, respectively, i.e. we have:

$$
g(j \omega):=R(\omega)+j I(\omega), \quad \omega \in \mathbf{R} .
$$

By using (44) in (43), we conclude that either $c_{1}=0$, or the following holds:

$$
\begin{gathered}
\omega \cos \omega-\omega I(\omega) \sin \omega=0 \\
\omega R(\omega) \sin \omega=0 .
\end{gathered}
$$

Assume that $c_{1} \neq 0$. Since $\lambda=0$ is not an eigenvalue of $L$, (see the proof of part (ii) of Theorem 1), we have $\omega \neq 0$. Also by (7), [see also (11)], $R(\omega)>\gamma>0, \forall \omega \in \mathbf{R}$. Hence, (46) implies that $\sin \omega=0$. Since $g(s)$ has at most a simple pole at $s=0, \omega I(\omega)$ is bounded at $\omega=0$. Hence, (45) implies that $\cos \omega=0$, which yields a contradiction since we also have $\sin \omega=0$. Therefore, in (43) we must have $c_{1}=0$, which, by (42) will yield to the trivial solution. This shows that the imaginary axis does not belong to the spectrum of $L$, hence must belong to the resolvent of $L$, (note that $L$ has a point spectrum).

To prove the estimate (41), we first solve (24)-(27) for $(f h r)^{\mathrm{T}} \in \mathscr{H}$ and $\lambda=j \omega, \omega \in \mathbf{R}$. By (29), the solution $u$ which satisfies $u(0)=0$ is given as:

$$
u(x)=j c_{1} \sin \omega x-\frac{1}{\omega} \int_{0}^{x}(h(s)+j \omega f(s)) \sin (x-s) \mathrm{d} s
$$$$
x \in(0,1),
$$ 
where $c_{1}$ is given by [see (32)]

$$
\begin{aligned}
& \int_{0}^{1}(h(s)+j \omega f(s))(\cos \omega(1-s) \\
& +j g(j \omega) \sin \omega(1-s)) \mathrm{d} s \\
& +\left[g(j \omega)-\frac{k}{j \omega}\right] f(1)-c^{\mathrm{T}}(j \omega I-A)^{-1} r \\
& j \omega \cos \omega-\omega g(j \omega) \sin \omega
\end{aligned}
$$

and $g(j \omega)$ is given by (8); $v(x)$ and $w$ can be found from (24) and (26) as:

$$
\begin{array}{r}
v(x)=-\omega c_{1} \sin \omega x-j \int_{0}^{x}(h(s)+j \omega f(s)) \sin \omega(x-s) \mathrm{d} s-f(x) \\
x \in(0,1) \\
w=(j \omega I-A)^{-1}[j \omega u(1) b+r-f(1) b] .
\end{array}
$$

Using integration by parts, we obtain:

$\int_{0}^{x} f(s) \cos \omega(x-s) \mathrm{d} s=\frac{1}{\omega} \int_{0}^{x} f^{\prime}(s) \sin \omega(x-s) \mathrm{d} s, \quad x \in(0,1)$

$$
\int_{0}^{x} f(s) \sin \omega(x-s) \mathrm{d} s=\frac{1}{\omega} f(x)-\frac{1}{\omega} \int_{0}^{x} f^{\prime}(s) \cos \omega(x-s) \mathrm{d} s
$$$$
x \in(0,1) \text {. }
$$

By differentiating (47) with respect to $x$, using (51) and Jensen's inequality [see Royden (1968, p. 110)] we obtain:

$$
\int_{0}^{1}\left|u_{x}\right|^{2} \mathrm{~d} x \leq 3\left(\omega^{2} c_{1}^{2}+\int_{0}^{1} h^{2}(s) \mathrm{d} s+\int_{0}^{1}\left(f^{\prime}\right)^{2}(s) \mathrm{d} s\right)
$$

Similarly, by using (52) and Jensen's inequality in (49), we obtain:

$$
\int_{0}^{1}|v|^{2} \mathrm{~d} x \leq 3\left(\omega^{2} c_{1}^{2}+\int_{0}^{1} h^{2}(s) \mathrm{d} s+\int_{0}^{1}\left(f^{\prime}\right)^{2}(s) \mathrm{d} s\right) .
$$

Also, by using (47), (52) and Jensen's inequaltiy in (50) we obtain:

$$
\begin{aligned}
\|w\|^{2} \leq & K_{1}\left\|(j \omega I-A)^{-1}\right\|^{2}\left(\omega^{2} c_{1}^{2}+\int_{0}^{1} h^{2}(s) \mathrm{d} s\right. \\
& \left.+\int_{0}^{1}\left(f^{\prime}\right)^{2}(s) \mathrm{d} s+\|r\|^{2}\right),
\end{aligned}
$$

where $K_{1}=4 \max \left\{\|b\|^{2}, 1\right\}$.

To obtain a bound on $\omega c_{1}$ for large $\omega$, we consider the denominator of (48):

$$
\begin{aligned}
D(\omega)^{2} & =|j \cos \omega-g(j \omega) \sin \omega|^{2} \quad \forall \omega \in \mathbf{R} \\
& \geq \gamma^{2} \sin ^{2} \omega+\cos ^{2} \omega-2 I(\omega) \sin \omega \cos \omega,
\end{aligned}
$$

where we use (44) and (11). (Here $|\cdot|$ denotes the absolute value of a complex number.) Since $I(\omega)$ decays at least as $O(1 / \omega)$ for large $\omega$ [see (8)], and since $\sin ^{2} \omega+\cos ^{2} \omega=1$, it follows from (56) that there exists a constant $K_{2}>0$ such that for $\omega$ sufficiently large, we have $D(\omega) \geq K_{2}$. By using this result, (51) and (52) in (48), and by noting that both $|g(j \omega)|$ and $\left\|(j \omega I-A)^{-1}\right\|$ are bounded for large $\omega$, we obtain the following estimate for large $\omega$ :

$$
\left|\omega c_{1}\right|^{2} \leq K_{3}\left(\int_{0}^{1} h^{2}(s) \mathrm{d} s+\int_{0}^{1}\left(f^{\prime}\right)^{2}(s) \mathrm{d} s+\|r\|^{2}+f^{2}(1)\right)
$$

for some $K_{3}>0$.

We note that $\left\|(j \omega I-A)^{-1}\right\|$ decays at least as $O\left(\frac{1}{\omega}\right)$ for large $\omega$, and that

$$
\lambda_{\text {min }}(P)\|w\|^{2} \leq w^{\top} P w \leq \lambda_{\max }(P)\|w\|^{2}, \quad w \in \mathbf{R}^{n}, \quad \text { (58) }
$$

where $\lambda_{\min }(P)$ and $\lambda_{\max }(P)$ are the minimum and maximum eigenvalues of $P$, respectively. Hence, by using (22), (53)-(55) and (57)-(58), we obtain the following estimate for large $\omega$ :

$$
\begin{aligned}
& \int_{0}^{1} u_{x}^{2} \mathrm{~d} x+\int_{0}^{1} v^{2} \mathrm{~d} x+u^{2}(1)+w^{\mathrm{T}} P w \\
& \leq K\left(\int_{0}^{1} h^{2}(s) \mathrm{d} s+\int_{0}^{1}\left(f^{\prime}\right)^{2}(s) \mathrm{d} s+\|r\|^{2}+f^{2}(1)\right)
\end{aligned}
$$

for some $K>0$.

Since the imaginary axis belongs to the resolvent set $\rho(L)$ of the operator $L$, and since for each $\lambda \in \rho(L),(\lambda I-L)^{-1}$ is compact, it follows that for any $\Omega<\infty$

$$
\sup _{\omega \leq \Omega}\left\|(j \omega I-L)^{-1}\right\|<\infty .
$$

Hence, from (59) and (60), we conclude that the estimate given by (41) holds. Therefore, by Theorem 2, we conclude that the $C_{0}$-semigroup $T(t)$ generated by the operator $L$ is exponentially stable, that is (40) holds.

\section{Simulation results}

In this section we show the effect of the proposed control law given by (4) and (5) on the solutions of the system given by (1)-(3), by means of some numerical simulation results. We assume that the controller output [i.e. (5)] is corrupted by a disturbance $d(t)$ as follows:

$$
f(t)=c^{\mathrm{T}} w(t)+d y_{t}(1, t)+k y(1, t)+d(t) .
$$

For the compensator transfer function $g(s)$, we choose the following [see (8)]

$$
g(s)=d+\frac{k}{s}+\frac{k_{1} s+k_{2}}{s^{2}+2 \xi \omega_{0} s+\omega_{0}^{2}} .
$$

Simple calculations show that when $d>0, k_{1} \geq 0, k_{2} \geq 0$, $\xi>0$ and $\omega_{0}>0,(11)$ is satisfied if $2 \xi \omega_{0} k_{1}-k_{2} \geq 0$.

For simulations, we first obtain a state-space representation [i.e. (4), (5)], for the compensator given by (62). For this purpose we choose the well-known controllable canonical representation of $g(s)$. Then we use the finite difference technique with $N$ point spatial discretization, approximating the spatial derivatives by using a central difference formula, see Greenspan and Casulli (1988). The resulting equations can be written in the form:

$$
\dot{z}=F z+b d(t)
$$

where the vector $z$ contains the displacements and the velocities at $N$ points, and $n$ compensator states. Hence, $F \in \mathbf{R}^{m \times m}$ and $b \in \mathbf{R}^{m}, m=2 N+n$. This equation is then simulated by using a trapezoidal type algorithm. In the simulations we choose $N=50$. As for the initial conditions we choose the initial displacement along the first mode of the uncontrolled system [i.e. (1)-(3) with $f(t) \equiv 0$ ], and we set:

$$
y(x, 0)=-0.5 \sin (0.5 \pi x), \quad y_{t}(x, 0)=0, \quad 0 \leq x \leq 1 .
$$

Initial conditions for the compensator states are set to zero.

For the disturbance $d(t)$, we choose four different types of waveforms which are given below.

(i) $d(t)=\cos 10 t$.

For this disturbance, we use the following sets of parameters, [see (62)]:

Case i.1. $d=1, k=0, k_{1}=0, k_{2}=0$.

Case i.2. $d=10, k=0, k_{1}=0, k_{2}=0$.

Case i.3. $d=1, k=0, k_{1}=100, k_{2}=100, \xi=0.1, \omega_{0}=10$.

Case i.4. $d=0.1, k=0, k_{1}=100, k_{2}=100, \xi=0.1, \omega_{0}=10$.

The resulting end point positions $y(1, t)$ for the Cases i.1-i.2 and i.3-i.4 are shown in Figs 1 and 2 , respectively. Obviously, with $k_{1}=k_{2}=0$, the controller given by $(62)$ reduces to the controller given by (6). It is known that in this case for $k=0, d \neq 1$, the decay rate $\delta$ [see (40)] can be given as $\delta=0.5 \ln |(1-d) /(1+d)|$, see e.g. Rideau (1985). Moreover, for $d=1$, it is known that the solutions decay to zero in finite time, see e.g. Majda (1975). Hence, from an eigenvalue placement point of view, the parameter values

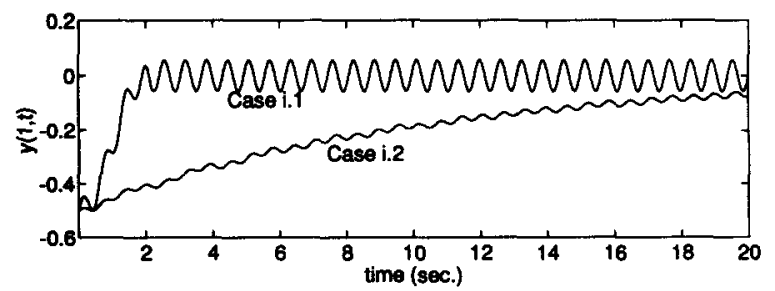

FIG. 1. Deflection $y(1, t)$ for the Cases i.1 and i.2. 


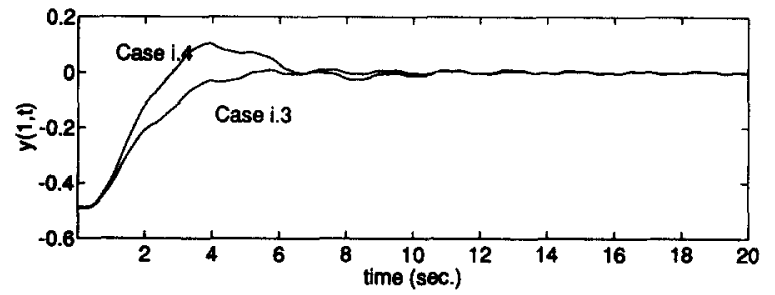

Fig. 2. Deflection $y(1, t)$ for the Cases i.3 and i.4.

chosen in the Case i.1 are optimal. Nevertheless, when the disturbance term given above is present, the solution $y(1, t)$ shows relatively large oscillations, as can be seen in Fig. 1. Increasing $d$ might yield better disturbance rejection, but as can be seen in the Fig. 1, this slows down the system response. Decreasing $d$ also results in very oscillatory behaviour in $y(1, t)$.

To decrease the rise time, hence to force the system to react fast, we choose a dynamic compensator $g(s)$ given by (62) with the parameter values given in the Cases i.3 and i.4. The resonant frequency $\omega_{0}$ is chosen to match the disturbance frequency and $k_{2}=100$ is chosen so that $\mathscr{R} e(g(j 0))=1+d$, hence even for $d=0$, we have $\mathscr{R e}(g(j 0))=1$. From Fig. 2 we see that the response is fast and the disturbance rejection is better. These simulations suggest that such a behaviour cannot be obtained by simply using a non-dynamic compensator [see (6)]. Also, as can be seen in Fig. 2, the decrease in $d$ results in an increase in the overshoot of the response.

(ii) $d(t)=0.1+\cos 10 t$.

The purpose of this choice for the disturbance is to show the effect of $k$ when a constant disturbance is present. For this disturbance $d(t)$, we choose the following sets of paramaters:

Case ii.1. $d=1, k=0, k_{1}=0, k_{2}=0$.

Case ii.2. $d=5, k=5, k_{1}=0, k_{2}=0$

Case ii.3. $d=1, k=0, k_{1}=100, k_{2}=100, \xi=0.1, \omega_{0}=10$.

Case ii.4. $d=1, k=5, k_{1}=100, k_{2}=100, \xi=0.1, \omega_{0}=10$.

The resulting end point position $y(1, t)$ for the Cases ii.1-ii.2 and ii.3-ii.4 are shown in Figs 3 and 4, respectively. These simulations suggest that in the presence of a constant disturbance, better disturbance rejection may be obtained for $k>0$. This is not too surprising since the term $k$ in (5) [or in (62)] corresponds to an integral term, and it is well-known in the classical feedback theory that the integral feedback terms yield better disturbance rejection in the presence of constant disturbances.

(iii) In this simulation we choose $d(t)$ as a periodic ramp function with a period $T=0.2 \mathrm{~s}$. More precisely we choose:

$$
d(t)=-1+t, \quad 0 \leq t \leq 0.2 \mathrm{~s}
$$

and $d(t+n T)=d(t)$ for $0 \leq t \leq 0.2 \mathrm{~s}$ and $n=1,2, \ldots$ The Fourier series expansion of this waveform contains infinitely many terms, which contain integer multiples of the fundamental frequency $5 \mathrm{~Hz}$. For this disturbance we choose the following sets of parameters:

Case iii.1. $d=1, k=0, k_{1}=0, k_{2}=0$.

Case iii.2. $d=5, k=0, k_{1}=0, k_{2}=0$.

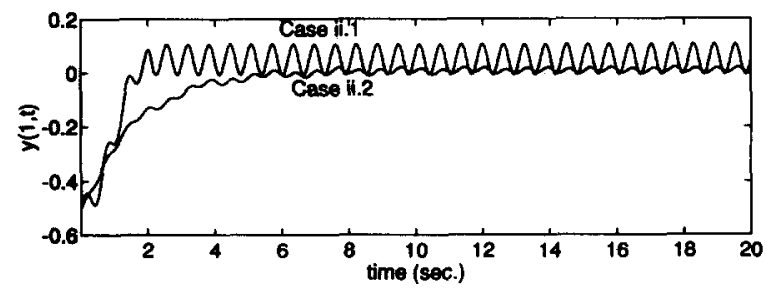

FIG. 3. Deflection $y(1, t)$ for the Cases ii.1 and ii.2.

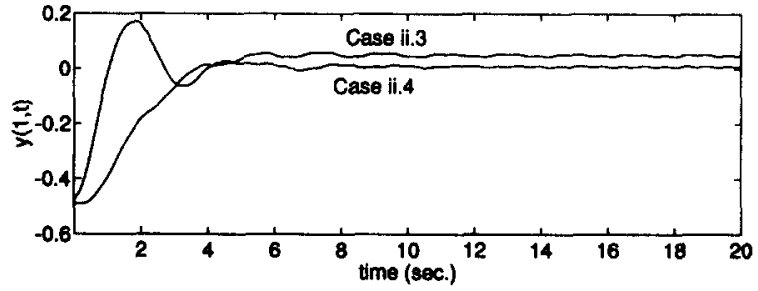

FIG. 4. Deflection $y(1, t)$ for the Cases ii.3 and ii.4.

Case iii.3. $d=1, k=0, \quad k_{1}=100 \pi^{2}, \quad k_{2}=100 \pi^{2}, \quad \xi=0.1$, $\omega_{0}=10 \pi$.

Case iii.4. $d=5, k=0, k_{1}=100 \pi^{2}, \quad k_{2}=100 \pi^{2}, \xi=0.1$, $\omega_{0}=10 \pi$.

The resulting end point positions $y(1, t)$ are shown in Figs 5 and 6 . In the Cases iii. 3 and iii. 4 we choose $\omega_{0}=10 \pi$ to match the fundamental frequency of the periodic ramp function. These simulations suggest that when the disturbance is a periodic waveform, one could use the dynamic compensator of the form given by (62) to get better disturbance rejection, provided that the resonance frequency $\omega_{0}$ is chosen to match the fundamental frequency of the periodic waveform. However, if the higher harmonics of the disturbance also have energy comparable to the energy of the fundamental harmonic, then this scheme may result in poor disturbance rejection.

(iv) In this simulation, to see the effect of random disturbances, we add a random signal with normal distribution, zero mean and unit variance to the periodic ramp function given by (63). For this disturbance, we choose the following sets of parameters:

Case iv.1. $d=1, k=0, k_{1}=0, k_{2}=0$

Case iv.2. $d=5, k=0, k_{1}=0, k_{2}=0$

Case iv.3. $d=1, \quad k=0, \quad k_{1}=100 \pi^{2}, \quad k_{2}=100 \pi^{2}, \quad \xi=0.1$, $\omega_{0}=10 \pi$.

Case iv.4. $d=5, \quad k=0, \quad k_{1}=100 \pi^{2}, \quad k_{2}=100 \pi^{2}, \quad \xi=0.1$, $\omega_{0}=10 \pi$.

The resulting end point position $y(1, t)$ for these cases are shown in Figs 7 and 8 . As can be seen from these figures, the dynamic compensator used in the Case iv. 3 gives better results as compared to the standard non-dynamic one used in the case iv.1.

These simulations suggest that, by using dynamic compensators, one may improve the system response in the case where the system is subject to disturbance. Moreover, once the compensator transfer function is parameterized [see (62)], optimum values for these parameters to shape the system response may be obtained (e.g. to decrease the overshoot, to decrease the rise time, etc.). However, this point needs further investigation.

\section{Conclusion}

In this note, we consider a linear time-invariant distributed parameter system described by a one-dimensional wave equation in a bounded domain (e.g. string, cable). We assumed that the system is clamped at one end and boundary control input is applied at the other end [see (1)-(3)]. To stabilize the system, we proposed a finite dimensional dynamic controller [see (4), (5)]. The transfer function of the

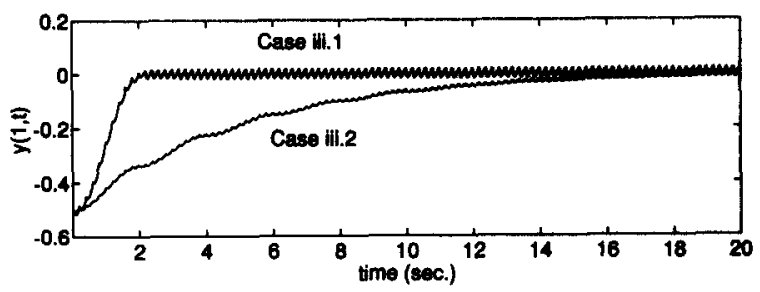

FIG. 5. Deflection $y(1, t)$ for the Cases iii.1 and iii.2. 


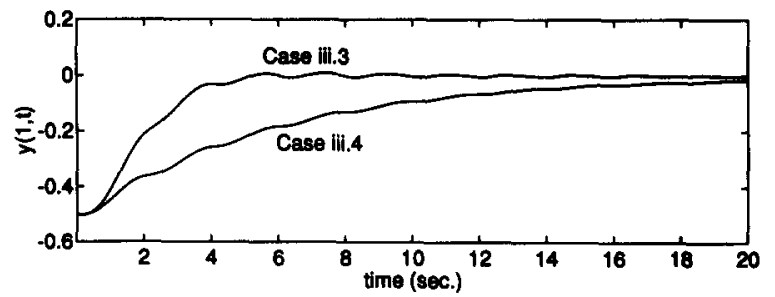

FIG. 6. Deflection $y(1, t)$ for the Cases iii.1 and iii.4.

controller is restricted to be a positive real function [see the Assumptions 1-3, and (11)]. This class of controllers can also be used in the stabilization of a Euler-Bernoulli beam, see Morgul (1992a). We proved that if the transfer function of the controller is strictly proper, then the resulting closed-loop system is asymptotically stable; moreover following Helmicki et al. (1991), one can show that the stability in this case cannot be exponential, (see Remark 2). We also show that if the controller transfer function is proper but not strictly proper, then the resulting closed-loop system is exponentially stable. We also presented some simulation results in the case where the controller output is corrupted by a disturbance. These results suggest that it may be possible to shape the system response (e.g. end point position) by using suitable dynamic controller transfer functions and that the dynamic controllers perform better than the standard non-dynamic ones [cf. (6) and (62)]. However this point requires further investigation.

The class of stabilizing controllers proposed here is quite large and covers some previously proposed controllers as a special case [see (6)]. This introduces extra degrees of freedom in designing controllers, which could be exploited in solving a variety of control problems, such as disturbance rejection, pole assignment, etc., while maintaining stability. Obviously the dynamic controllers offer more degrees of freedom than the standard non-dynamic one to solve these problems. Preliminary simulation studies show that by using the proposed controller, it could be possible to change the spectrum of the system given by (1)-(3) over a specified frequency range, while not disturbing the rest of the spectrum very much. This may not be obtained if one uses a constant feedback, since in this case the whole spectrum is affected uniformly. This point, and other applications of the proposed dynamic controller, require further investigation.

It has been claimed that all physical systems show strictly proper behaviour in the sense that they do not respond instantaneously to applied inputs, see Helmicki et al. (1991). From this point of view, the non-dynamic controller given by (6) may not represent a physical device unless $d=0$. However if $d=0$, the system given by (1)-(3) and (6) is not asymptotically stable. On the other hand, the dynamic controllers given by (4), (5), with $d=0$, have strictly proper transfer functions. Moreover, if these controllers satisfy the Assumptions 1-3, then by Theorem 1 the resulting closed-loop system is asymptotically stable. This argument shows the practical importance of the results presented in this paper.

We also note that, the transfer function of the controller is allowed to be a strictly proper function. In this case, one can show that the open-loop map of the system is also strictly proper (see the Remark 2 ). This is certainly a desirable

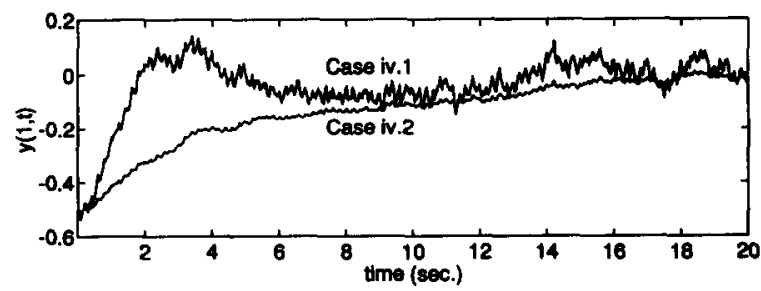

FIG. 7. Deflection $y(1, t)$ for the Cases iv.1 and iv.2.

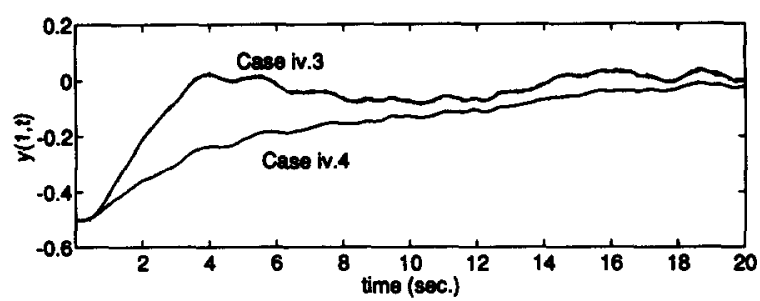

FIG. 8. Deflection $y(1, t)$ for the Cases iv. 3 and iv.4.

property in proving certain stability robustness results. However, the obtained stability is only asymptotic in this case, and not exponential. This shows that in constructing an algebraic framework for studying the stability of certain distributed parameter systems, to include the asymptotic stability in the stability definition, rather than exponential stability, might be a proper choice, see Callier and Wilkin (1986) and Helmicki et al. (1991). However, this point also needs further investigation.

\section{References}

Bardos, C., G. Lebeau and J. Rauch (1992). Sharp sufficient conditions for the observation, control and stabilization of waves from the boundary. SIAM J. Contr. Optim., 30, 1024-1066.

Callier, F. M. and J. Wilkin (1986). Distributed system transfer functions of exponential order. Int. J. Contr., 43, 1353-1373.

Chen, G..(1979). Energy decay estimates and exact boundary value controllability for the wave equation in a bounded domain. J. Math. Pure Appl., 58, 249-273.

Chen, G., M. C. Delfour, A. M. Krall and G. Payre (1987a) Modelling, stabilization and control of serially connected beams. SIAM J. Contr. Optim., 25, 526-546.

Chen, G., S. G. Krantz, D. W. Ma, C. E. Wayne and H. H. West (1987b). The Euler-Bernoulli beam equation with boundary energy dissipation. In S. J. Lee (Ed.) Operator Methods for Optimal Control Problems, also in Lecture Notes in Pure and Applied Mathematics Series, pp. 67-96. Marcell-Dekker, NY.

Conrad, F. and B. Rao (1993). Decay of solutions of the wave equation with nonlinear boundary control. In R. F. Curtain (Ed.) Analysis and Optimization of Systems: State and Frequency Domain Approaches for Infinite Dimensional Systems. Lecture Notes in Control and Information Sciences, 185, 512-524.

Dafermos, C. M. and M. Slemrod (1973). Asymptotic behaviour of nonlinear contraction semigroups. $J$. of Functional Analysis, 13, 97-106.

d'Andrea-Novel, B., F. Boustany and F. Conrad (1990) Control of an overhead crane: stabilization of flexibilities. IFIP Boundary Control and Boundary Variations Workshop, Sophia-Antipolis, France.

Datko, R. (1988). Not all feedback stabilized hyperbolic systems are robust with respect to small time delays in their feedbacks. SIAM J. Contr. Optim., 26, 697-713.

Gearhart, L. (1978). Spectral theory for contraction semigroups on Hilbert space. Trans. of the Am. Math. Soc., 236, 385-394.

Greenspan, D. and V. Casulli (1988). Numerical Analysis for Applied Mathematics, Science and Engineering. AddisonWesley, Reading, MA.

Hale, J. K. (1969). Dynamical systems and stability. J. of Mathematical Analysis an Applications, 26, 39-59.

Helmicki, A. J. (1988). Practical considerations in the use of distibuted parameter systems in modelling: a control point of view. M.Sc. Thesis, Rensselear Polytechnic Institute, Troy, New York.

Helmicki, A. J., C. A. Jacobson and C. N. Nett (1991) Ill-posed distributed parameter systems: a control viewpoint. IEEE Trans. Autom. Control., 36, 1053-1057.

Huang, F. L. (1985). Characteristic conditions for exponential stability of linear dynamical systems in Hilbert spaces. Annales Diff. Equations, 1, 43-53. 
Kato, T. (1980). Perturbation Theory for Linear Operators (2nd edition). Springer-Verlag, NY.

Komornik, V. and E. Zuazua (1990). A direct method for the boundary stabilization of the wave equation. J. Math. Pure Appl., 69, 33-54.

Lagnese, J. (1983). Decay of solutions of wave equations in a bounded domain with boundary dissipation. $J$. of Differential Equations, 50, 163-182.

Lagnese, J. (1989). Boundary stabilization of thin plates. SIAM Studies in Applied Mathematics, 10, SIAM, Philadelphia, PA.

Lasiecka, I. (1989). Stabilization of wave and plate-like equations with nonlinear dissipation on the boundary. $J$. Diff. Eqns., 79, 340-381.

Lasiecka, I. and R. Triggiani (1987). Uniform exponential energy decay of the wave equation in a bounded domain with $L_{2}\left(0: \infty ; L_{2}(\Gamma)\right)$-feedback control in the Dirichlet boundary conditions. J. Diff. Eqns., 66, 340-390.

Lions, J.-L. (1988). Exact controllability, stabilization and perturbations for distributed parameter systems. SIAM Review, 30, 1-68.

Majda, A. (1975). Disappearing solutions for the dissipative wave equation. Indiana Univ. Math. J., 24, 11191133.

Meirovitch, L. (1967). Analytical Methods in Vibration. Macmillan, NY.

Morgül, Ö. (1990). Control and stabilization of a flexible beam attached to a rigid body. Int. J. Contr., 51, 11-33.

Morgül, Ö (1991a). Orientation and stabilization of a flexible beam attached to a rigid body: planar motion. IEEE Trans. on Autom. Contr., 36, 953-963.

Morgül, Ö (1991b). Boundary control of a Timoshenko beam attached to a rigid body: planar motion. Int. J. Contr., 54, $763-791$

Morgül, Ö (1992a). Dynamic boundary control of a Euler-Bernoulli beam. IEEE Trans. on Autom. Contr., 37, 639-642.

Morguil, Ö. (1992b). Dynamic boundary control of the Timoshenko beam. Automatica, 28, 1255-1260.

Pazy, A. (1983). Semigroups of Linear Operators and Applications to Partial Differential Equations. SpringerVerlag, NY.

Prüss, J. (1984). On the spectrum of $C_{0}$-semigroups. Trans. of the Am. Math. Soc., 284, 847-857.

Rideau, P. (1985). Controle d'un assemblage de poutres flexibles par des capteurs-actionneurs ponctuels: étude du spectre du système. Thése, L'Ecole Nationale Superieriure des Mines de Paris, Sophia-Antipolis, France.

Royden, H. L. (1968). Real Analysis (2nd edition). MacMillan, NY

Saperstone, S. (1981). Semidynamical Systems in Infinite Dimensional Spaces. Springer-Verlag, NY.

Slemrod, M. (1976). Stabilization of boundary control systems. J. of Differential Equations, 22, 402-415.

Slotine, J. J. E. and W. Li (1991). Applied Nonlinear Control. Prentice-Hall, Englewood Cliffs, NJ.

Tanabe, H. (1979). Equations of Evolution. Pitman, London. 